\title{
Nota sobre rotação finita e vetor
}

\author{
Note on finite rotation and vector \\ M.T. Thomaz*10 \\ ${ }^{1}$ Universidade Federal Fluminense, Niterói, RJ, Brasil.
}

Recebido em 10 de Julho de 2020. Aceito em 27de Julho de 2020.

\begin{abstract}
Quando estudamos o movimento de rotação de corpos rígidos precisamos de uma cinemática de rotação que descreva este tipo de deslocamento que os corpos podem ter. É dito nos livros textos que: "...não podemos identificar rotações finitas com vetores." Esta nota usa uma demonstração algébrica simples para mostrar a validade dessa afirmação no caso do movimento angular finito de uma partícula e entender porque apesar desta impossibilidade podemos associar um vetor a uma variação angular infinitesimal, sem qualquer conflito.

Palavras-chave: Rotação finita, rotação infinitesimal, vetor, vetor deslocamento angular.
\end{abstract}

When we study the rotation movement of rigid bodies we need a rotation kinematics that describes this type of displacement that the bodies can have. It is said in the textbooks that: "... we cannot identify finite rotations with vectors." This note uses a simple algebraic demonstration to show the validity of this statement in the case of finite angular movement of a particle and to understand why despite this impossibility we can associate a vector with an infinitesimal angular variation, without any conflict.

Keywords: Finite rotation, infinitesimal rotation, vector, angular displacement vector.

Existem questões que nos perseguem pela vida a fora. A impossibilidade de associar um vetor para representar a rotação finita de uma partícula, ou de um corpo extenso, era aceita por mim por estar nos livros textos [1, p. 65][2]. As explicações dessa impossibilidade sempre me pareceu conflitante com o fato das rotações infitesimais poderem ser representadas por vetores. As rotações finitas de qualquer corpo podem ser obtidas a partir de inúmeras rotações infinitesimais. Como entender que ambas as afirmações sobre a relação ou não entre vetores e rotações infinitesimais e finitas estão corretas?

Vejamos rapidamente o argumento similar ao apresentado nos livros textos [1, p. 65] e [2]: considere um objeto macroscópico, por exemplo uma boneca russa, uma matriosca. Realizamos duas rotações de $90^{\circ}$ nesta boneca em torno dos eixos $y$ e $z$. As duas rotações consecutivas realizadas na matriosca são no sentido anti-horário. Para mostrar os efeitos dessas rotações fotografamos a posição da boneca em relação aos eixos xyz quando mudamos a ordem em que as duas rotações são realizadas sobre ela. A matriosca está na mesma posição inicial nas Figs. 1a e $2 \mathrm{a}$.

A Fig.1b mostra a matriosca após ela sofrer uma uma rotação de $90^{\circ}$, no sentido anti-horário, em torno do eixo z. Obtemos a Fig.1c girando a boneca na Fig.1b de um ângulo de $90^{\circ} \mathrm{em}$ torno do eixo y no sentido anti-horário.

A Fig.2b é obtida da Fig.2a rodando a boneca de $90^{\circ}$ no sentido anti-horário em torno do eixo $y$. Finalmente

*Endereço de correspondência: mtt@if.uff.br rodamos a matriosca mostrada na Fig.2b de um ângulo de $90^{\circ}$ no sentido anti-horário em torno do eixo $z$. (a)

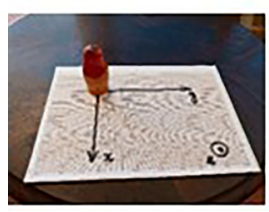

(b)

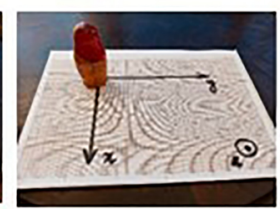

(c)

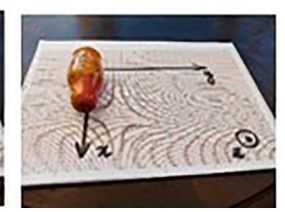

Figura 1: As posições da boneca matriosca quando ela sofre duas rotações de $90^{\circ}$ consecutivas, no sentido anti-horário, em torno dos eixos $z$ e $y$. a) posição inicial da boneca; $b$ ) rotação de $90^{\circ}$ no sentido anti-horário em torno do eixo $z ; c$ ) rotação também de $90^{\circ}$ no sentido anti-horário em torno do eixo $y$. (a)

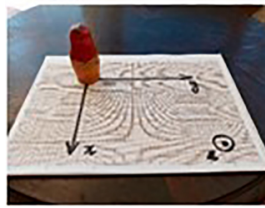

(b)

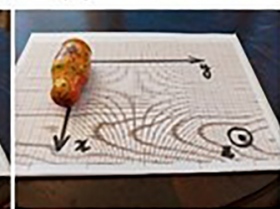

(c)

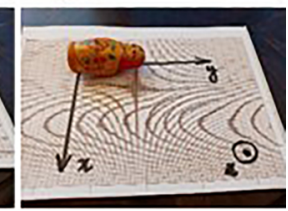

Figura 2: As posições da boneca matriosca quando ela sofre duas rotações de $90^{\circ}$ consecutivas, no sentido anti-horário, em torno dos eixos $z$ e $y$ em ordem inversa das Figs.1. a) mesma posição inicial da boneca que na Fig.1a; $b$ ) rotação de $90^{\circ}$, no sentido anti-horário, em torno do eixo $y ; c$ ) rotação também de $90^{\circ}$ em torno do eixo $z$ no sentido anti-horário. 
Para obter as Figs.1c e 2c realizamos as mesmas rotações em torno dos eixos $y$ e $z$, mas em ordens diferentes. Comparando essas duas figuras verificamos que elas levam a posições finais diferentes da boneca. Assim se associamos a cada rotação finita (ângulo de $90^{\circ}$, medido em radianos) um vetor paralelo ao eixo em torno do qual a matriosca é girada, a soma vetorial desses duas rotações deveria me dar um vetor que seria perpendicular ao plano que corresponderia levar a boneca da posição inicial a posição final. As Figs.1c e 2c nos mostram que a ordem com que somamos vetorialmente os vetores ligados as rotações não comutam entre si[1, p. 65]. No entanto, uma das propriedades de vetores é que a soma vetorial de dois vetores não depende da ordem em que eles são somados. Desta forma os livros textos [1, p. 65][ 2] nos mostram, usando objetos extensos, que não podemos representar deslocamentos angulares finitos por vetores.

Será que podemos usar partículas para mostrar esse resultado de que rotações finitas não podem ser descritas por vetores? Na discussão que segue consideramos uma partícula cuja a posição é rodada de dois ângulos finitos em torno de 2 eixos ortogonais entre si. Desejamos verificar se essas rotações da partícula podem ser descritas por vetores ou não. Devido a natureza cinemática do problema tratado aqui, não atribuimos outras propriedades a esse corpo pontual, tais como: massa, velocidade, etc...

Quando temos uma partícula percorrendo um movimento circular sobre uma superfície plana, temos uma única direção privilegiada nesta situação física: a direção perpendicular a superfície sobre a qual a partícula se desloca. Vamos assumir que podemos atribuir um vetor $\vec{\Phi}$ a rotação finita $\Phi$, medida em radianos, quando a partícula se desloca entre as posições $P_{1}$ e $P_{2}$ sobre a superfície plana $\boldsymbol{S}$. O vetor $\vec{\Phi}$ tem módulo igual a $|\Phi|$, e sua direção é paralela a reta $n$ perpendicular ao plano $\boldsymbol{S}$, veja a Fig.3. O sentido do vetor $\vec{\Phi}$ é dado pela regra da mão direita quando os dedos dessa mão vão se fechando ao percorrer a trajetória da partícula.

Se os vetores associados aos deslocamentos angulares dos corpos estão bem definidos, então se a partícula realiza rotações consecutivas, inclusive com rotações em planos diferentes, o vetor resultante $\vec{\Phi}$ que descreve a rotação da partícula da sua posição inicial até a sua posição final, tem que satisfazer as seguintes propriedades:

1. o vetor resultante associado as rotações satisfaz a regra do paralelogramo: o vetor resultante é igual a soma vetorial dos vetores associados a rotação da partícula em cada superfície plana;

2. o vetor resultante é perpendicular ao plano que contém a partícula nas suas posições inicial e final.

Vejamos se o vetor resultante $\vec{\Phi}$, associado ao movimento de rotação da partícula, que a leva da sua posição inicial a sua posição final através de movimentos de rotações finitas em planos distintos, satisfaz as condições 1 e 2 anteriores.

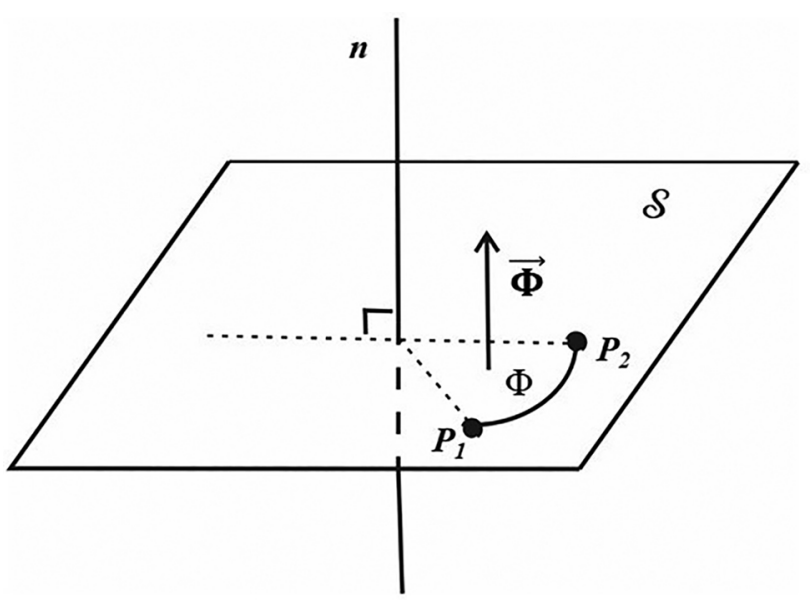

Figura 3: A partícula gira de um ângulo $\Phi$ ao variar a sua posição de $P_{1}$ para $P_{2}$ no plano $\boldsymbol{S}$. A reta $\boldsymbol{n}$ é perpendicular ao plano $S$. Associamos o vetor $\vec{\Phi}$ a rotação finita $\Phi$ que a partícula realiza neste plano durante seu movimento.

Para verificarmos se a definição do vetor $\vec{\Phi}$ tem realidade física, consideramos uma partícula cujos os movimentos de rotação em torno dos eixos $z$ e $y$ são mostrados na Fig.4.

Na posição inicial $P_{1}$ o vetor posição da partícula 1 é

$$
\overrightarrow{\boldsymbol{r}}_{P_{1}}=a \hat{\mathrm{i}}
$$

sendo $a \geq 0$ e $a \in \mathbb{R}$, e $\hat{\mathrm{i}}$ é o versor do eixo $x$.

A partir de sua posição inicial $P_{1}$, a partícula é girada de um ângulo $\varphi$ em torno do eixo $z$ mantendo uma distância $a$ da origem $\boldsymbol{O}$. O vetor $\vec{\varphi}$, associado a rotação da partícula em torno do eixo $z$, está desenhado na Fig.4b. Este vetor é igual a

$$
\vec{\varphi}=\varphi \hat{k}
$$

sendo $\hat{k}$ o versor do eixo $z$ e $0<\varphi<\pi / 2$.

$\mathrm{O}$ vetor posição da partícula quando ela ocupa a posição $P_{2}$ é

$$
\overrightarrow{\boldsymbol{r}}_{P_{2}}=\operatorname{acos}(\varphi) \hat{\mathrm{i}}+\operatorname{asen}(\varphi) \hat{\mathrm{j}}
$$

com $\hat{\mathrm{i}}$ e $\hat{\mathrm{j}}$ sendo os versores dos eixos $x$ e $y$, respectivamente.

A partícula é levada em seguida da posição $P_{2}$ para a posição $P_{3}$ através de uma rotação de um ângulo $\gamma$, no sentido horário em torno do eixo $y$. Durante essa rotação a distância da partícula ao eixo y é igual a $a \cos (\varphi)$. Apesar de $\gamma$ ser medido no sentido horário, aqui usamos a conveção de que $\gamma>0$, variando no intervalo $0<\gamma<\pi$. Durante esta rotação em torno do eixo $y$, a componente $y$ do vetor posição da partícula não varia e é igual a $y=\operatorname{asen}(\varphi)$.

$\mathrm{O}$ vetor posição que localiza a partícula em relação a origem $\boldsymbol{O}$ quando ela está em $P_{3}$ é

$\overrightarrow{\boldsymbol{r}}_{P_{3}}=a \cos (\varphi) \cos (\gamma) \hat{\mathrm{i}}+a \operatorname{sen}(\varphi) \hat{\mathrm{j}}+a \cos (\varphi) \operatorname{sen}(\gamma) \hat{k}$ 


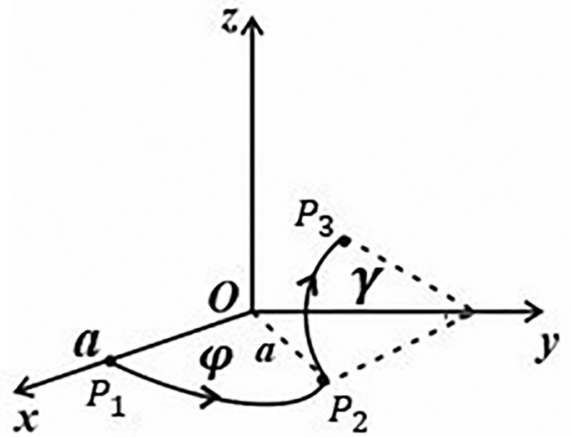

(a)

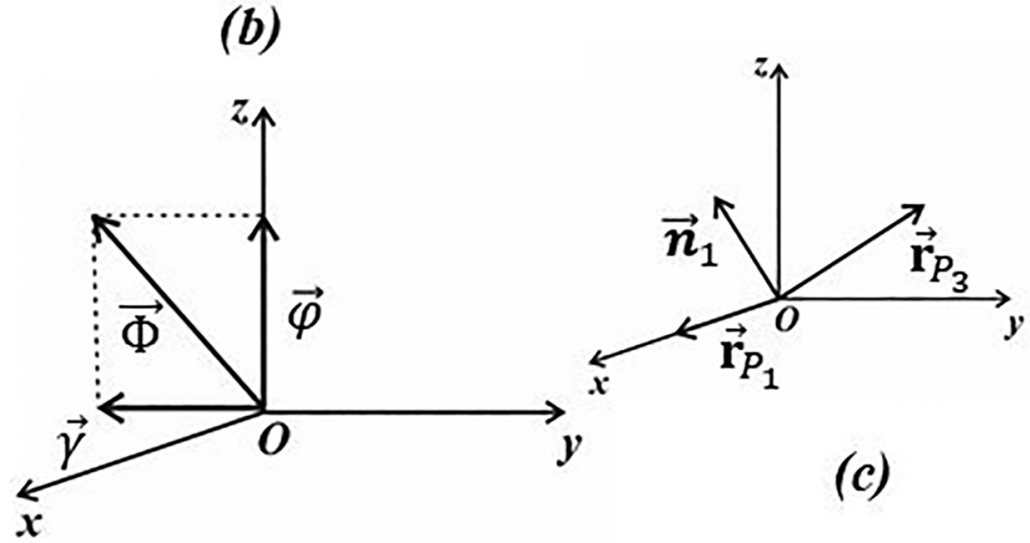

Figura 4: a) Apartícula está inicialmente sobre o eixo $x$ em $x=a$, posição $P_{1}$, e sofre uma rotação de um ângulo $\varphi$ em torno do eixo $z$ mantendo uma distância $a$ da origem $\boldsymbol{O}$, passando a ocupar a posição $P_{2}$. Em seguida ela roda de um ângulo $\gamma$ em torno do eixo $y$, terminando na posição $\left.P_{3} ; b\right)$ Vetores $\vec{\varphi}$ e $\vec{\gamma}$ estão associados respectivamente as rotações $\varphi$ e $\gamma$ em torno dos eixos $z$ e $y$; c) Os vetores $\overrightarrow{\boldsymbol{r}}_{P_{1}}$ e $\overrightarrow{\boldsymbol{r}}_{P_{3}}$ são os vetores posição da partícula em relação a origem $\boldsymbol{O}$ quando ela ocupa as posições $P_{1}$ e $P_{3}$, respectivamente. $\mathrm{O}$ vetor $\overrightarrow{\boldsymbol{n}}_{1}$ é perpendicular ao plano que contém os dois vetores posição nas posições inicial $\left(P_{1}\right)$ e final $\left(P_{3}\right)$.

O vetor $\vec{\gamma}$ que descreve a rotação da partícula indo da posição $P_{2}$ para a posição $P_{3}$ é

$$
\vec{\gamma}=\gamma(-\hat{\mathrm{j}}), \quad 0<\gamma<\pi .
$$

Então se $\vec{\Phi}$ é o vetor resultante associado a rotação que leva a partícula da sua posição inicial $P_{1}$ até a sua posição final $P_{3}$, pela condição 1 ele tem que ser igual a:

$$
\vec{\Phi}=\vec{\varphi}+\vec{\gamma} \Rightarrow \vec{\Phi}=-\gamma \hat{j}+\varphi \hat{k}
$$

Para que os vetores associados a rotações estejam bem definidos, o vetor resultante $\vec{\Phi}$ tem que ser prependicular ao plano que contém os vetores posição da partícula quando ela está localizada em $P_{1}$ e em $P_{3}$. Este plano também, contém o vetor $\Delta \overrightarrow{\boldsymbol{r}}_{P_{1}, P_{3}}$, que descreve a variação de posição da partícula entre as suas posições inicial e final. Este vetor é igual a:

$$
\begin{aligned}
& \Delta \overrightarrow{\boldsymbol{r}}_{P_{1}, P_{3}}=\overrightarrow{\boldsymbol{r}}_{P_{3}}-\overrightarrow{\boldsymbol{r}}_{P_{1}} \Longrightarrow \\
& \Longrightarrow \Delta \overrightarrow{\boldsymbol{r}}_{P_{1}, P_{3}}=\operatorname{acos}(\varphi) \cos (\gamma) \hat{\mathrm{i}} \\
& +a \operatorname{sen}(\varphi) \hat{\mathrm{j}}+\operatorname{acos}(\varphi) \operatorname{sen}(\gamma) \hat{k} .
\end{aligned}
$$

Para determinar se o vetor resultante $\vec{\Phi}$ é perpendicular ao plano que contém os vetores posição da partícula quando ela está em $P_{1}$ e $P_{3}$, basta calcular o seu produto escalar com o vetor $\Delta \overrightarrow{\boldsymbol{r}}_{P_{1}, P_{3}}$, isto é:

$$
\vec{\Phi} \cdot \Delta \overrightarrow{\boldsymbol{r}}_{P_{1}, P_{3}}=a(-\gamma \operatorname{sen}(\varphi)+\varphi \cos (\varphi) \operatorname{sen}(\gamma)),
$$

$\operatorname{com} 0<\varphi<\pi / 2$ e $0<\gamma<\pi$.

Para qualquer valor da coordenada $a \in \mathbb{R}_{+}$, no caso geral em que $\varphi$ e $\gamma$ assumem valores finitos o resultado 8 nos dá que o vetor $\vec{\Phi}$ não é perpendicular ao plano que contém os vetores posição da partícula na sua posição inicial $P_{1}$ e na sua posição final $P_{3}$, isto é, $\vec{\Phi} . \Delta \overrightarrow{\boldsymbol{r}}_{P_{1}, P_{3}} \neq 0$. Dessa forma o vetor resultante $\vec{\Phi}$ não satisfaz a condição
2, mostrando que a definição de vetor associado a rotações finitas não descreve a situação física e portanto não tem sentido definir este vetor.

No entanto, se a partícula sofre rotações infinitesimais, isto é, $\varphi=\Delta \varphi \rightarrow 0$ e $\gamma=\Delta \gamma \rightarrow 0$, em torno dos eixos $z$ e $y$, respectivamente, as seguintes aproximações são válidas:

$$
\begin{aligned}
\operatorname{sen}(\Delta \varphi) & =\Delta \varphi-\frac{(\Delta \varphi)^{3}}{6}+O\left(\Delta \varphi^{5}\right), \\
\cos (\Delta \varphi) & =1-\frac{(\Delta \varphi)^{2}}{2}+O\left(\Delta \varphi^{4}\right), \\
\operatorname{sen}(\Delta \gamma) & =\Delta \gamma-\frac{(\Delta \gamma)^{3}}{6}+O\left(\Delta \varphi^{5}\right) .
\end{aligned}
$$

Calculamos o produto escalar (8) até $4^{\mathrm{a}}$ ordem nas rotações infinitesimais $\Delta \varphi$ e $\Delta \gamma$, obtendo a aproximação,

$$
\begin{gathered}
\frac{1}{a} \Delta \vec{\Phi} \cdot \Delta \overrightarrow{\boldsymbol{r}}_{P_{1}, P_{3}} \approx-\frac{(\Delta \gamma) \cdot(\Delta \varphi)^{3}}{3} \\
-\frac{(\Delta \varphi) \cdot(\Delta \gamma)^{3}}{6} \underset{\substack{\Delta \varphi \rightarrow 0 \\
\Delta \gamma \rightarrow 0}}{\longrightarrow} 0,
\end{gathered}
$$

onde

$$
\Delta \vec{\Phi}=-\Delta \gamma \hat{\mathrm{j}}+\Delta \varphi \hat{k}
$$

com $\hat{j}$ e $\hat{k}$ sendo os versores dos eixos $y$ e $z$, respectivamente.

O resultado 10a nos mostra que no limite de rotações infinitesimais, $\Delta \varphi \rightarrow 0$ e $\Delta \gamma \rightarrow 0$, o vetor resultante $\Delta \vec{\Phi}$ associado a essas rotações infitesimais satisfaz a condição 2. O fato do resultado ser uma aproximação, e não um resultado exato é que explica que quando temos rotações finitas o vetor associado a essas rotações não pode ser 
definido, mesmo sabendo que uma rotação finita pode ser obtida por $N$ rotações infinitesimais com $N \rightarrow \infty$. No limite em que $N \rightarrow \infty$, o produto $N .\left(\vec{\Phi} \cdot \Delta \overrightarrow{\boldsymbol{r}}_{P_{1}, P_{3}}\right)$, com $\vec{\Phi} . \Delta \overrightarrow{\boldsymbol{r}}_{P_{1}, P_{3}}$ sendo aproximado pelo resultado $10 \mathrm{a}$, pode dar um resultado finito, mostrando que para rotações finitas o vetor associado pode não ser perpendicular ao plano que contém os vetores das posições inicial e final da partícula após rotações consecutivas em planos distintos.

Para concluirmos se os vetores associados a rotações infinitesimais descrevem corretamente ou não esse tipo de movimento, precisamos verificar se o vetor resultante $\vec{\Phi}$, dado pela equação (6), no caso de rotações infinitesimais $\Delta \varphi$ e $\Delta \gamma$ em torno dos eixos $z$ e $y$, respectivamente, depende da ordem em que essas rotações são realizadas pelo corpo pontual. Para isso, vamos fazer uma análise semelhante a que foi feita na referência [1 p. 276], só que agora para o caso em que o objeto que gira é um corpo pontual.

Calculamos a seguir o vetor normal ao plano que contém os vetores posição inicial da partícula na posição $P_{1}, \overrightarrow{\boldsymbol{r}}_{P_{1}}$, e na sua posição final $P_{3}, \overrightarrow{\boldsymbol{r}}_{P_{3}}$, na trajetória percorrida pela partícula na Fig.4a. Ambos os vetores estão desenhados na Fig.4c.

A equação (1) nos dá a expressão do vetor posição, $\overrightarrow{\boldsymbol{r}}_{P_{1}}$. $\mathrm{O}$ vetor posição da partícula em $P_{3}$ é obtido da Fig.4a, sendo igual a

$$
\begin{aligned}
& \overrightarrow{\boldsymbol{r}}_{P_{3}}=a \cos (\varphi) \cos (\gamma) \hat{\mathrm{i}}+a \operatorname{sen}(\varphi) \hat{\mathrm{j}} \\
& +a \cos (\varphi) \operatorname{sen}(\gamma) \hat{k}
\end{aligned}
$$

onde $a>0$ e $a \in \mathbb{R}, \varphi \in\left[0, \frac{\pi}{2}\right]$ e $\gamma \in[0, \pi]$.

Denominamos de $\overrightarrow{\boldsymbol{n}}_{1}$ o vetor perpendicular ao plano ao qual pertencem os vetores posição $\overrightarrow{\boldsymbol{r}}_{P_{1}}$ e $\overrightarrow{\boldsymbol{r}}_{P_{3}}$, desenhados na Fig.4c,

$$
\begin{aligned}
& \overrightarrow{\boldsymbol{n}}_{1}=\overrightarrow{\boldsymbol{r}}_{P_{1}} \times \overrightarrow{\boldsymbol{r}}_{P_{3}} \\
& =a^{2}(-\cos (\varphi) \operatorname{sen}(\gamma) \hat{\mathrm{j}}+\operatorname{sen}(\varphi) \hat{k}) .
\end{aligned}
$$

Voltamos a considerar a partícula na mesma posição inicial que na Fig.4a. Em seguida giramos este corpo de um ângulo $\gamma$ em torno do eixo $y$ no sentido horário e depois realizamos uma rotação $\varphi$ em torno do eixo $z$, como mostrado na Fig. 5a. A partícula ao rodar do ângulo $\varphi$ em torno do eixo $y$, ela se mantém a uma distância $a$ da origem $\boldsymbol{O}$. Durante a rotação do corpo do ângulo $\gamma$ em torno do eixo $z$, ele permanece a uma distância $\operatorname{acos}(\gamma)$ desse eixo.

O vetor $\overrightarrow{\boldsymbol{r}}_{P_{3}^{\prime}}$, que localiza a partícula na sua posição final $P_{3}^{\prime}$ e está desenhado na Fig.5a, tem a seguinte expressão,

$$
\begin{aligned}
& \overrightarrow{\boldsymbol{r}}_{P_{3}^{\prime}}=a \cos (\varphi) \cos (\gamma) \hat{\mathrm{i}} \\
& +a \operatorname{sen}(\varphi) \cos (\gamma) \hat{\mathrm{j}}+a \operatorname{sen}(\gamma) \hat{k},
\end{aligned}
$$

com $a>0$ e $a \in \mathbb{R}_{+}, \varphi \in\left[0, \frac{\pi}{2}\right]$ e $\gamma \in[0, \pi]$.

O vetor $\overrightarrow{\boldsymbol{n}}_{\mathbf{2}}$, desenhado na Fig.5b, é perpendicular ao plano que contém os vetores na posição inicial, $\overrightarrow{\boldsymbol{r}}_{P_{1}}$, e final da partícula, $\overrightarrow{\boldsymbol{r}}_{P_{3}^{\prime}}$. A sua expressão é igual a

$$
\begin{gathered}
\overrightarrow{\boldsymbol{n}}_{2}=\overrightarrow{\boldsymbol{r}}_{P_{1}} \times \overrightarrow{\boldsymbol{r}}_{P_{3}^{\prime}} \\
=a^{2}(-\operatorname{sen}(\gamma) \hat{\mathrm{j}}+\operatorname{sen}(\varphi) \cos (\gamma) \hat{k}) .
\end{gathered}
$$

Comparamos as expressões (12b) e (14b) dos vetores $\overrightarrow{\boldsymbol{n}}_{1}$ e $\overrightarrow{\boldsymbol{n}}_{2}$, verificamos que esses vetores são distintos no caso em que os ângulos $\varphi$ e $\gamma$ são finitos. A diferença entre esses vetores é que explica as posições diferentes da boneca matriosca nas Figs.1c e 2c. Ressaltamos que esses vetores deveriam corresponder ao vetor resultante $\vec{\Phi}$ em cada uma das situações físicas correspondentes as Figs. 4 e 5. Com os resultados (12b) e (14b) mostramos que o vetor resultante das duas rotações finitas consecutivas que o vetor resultante associado as essas rotações também não satisfaz a condição 1, uma vez que a expressão do vetor resultante $\vec{\Phi}$ dependeria da ordem em que as rotações finitas são realizadas pela partícula.

\section{(a)}

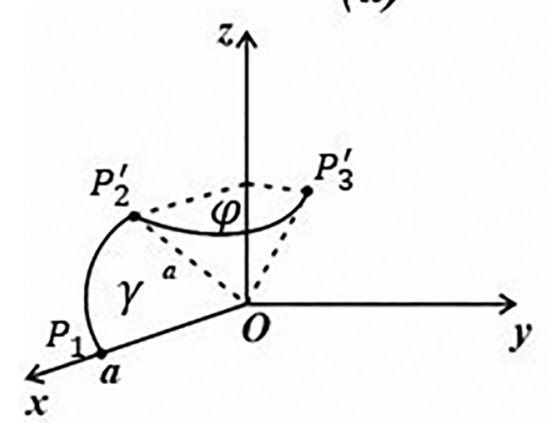

(b)

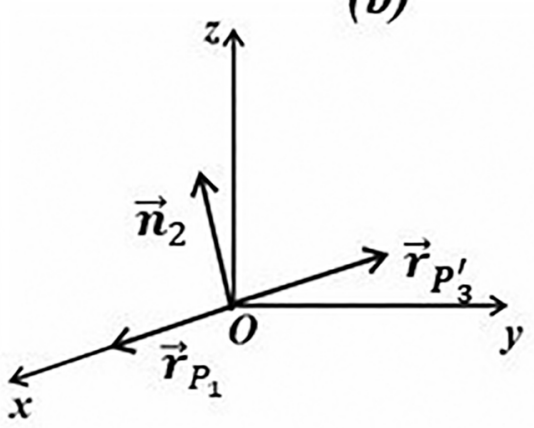

Figura 5: a) A partícula está na mesma posição inicial que na Fig.4a, em $x=a$, em $P_{1}$, quando ela é girada do ângulo $\gamma$ em torno do eixo $y$, alcançando a posição $P_{2}$. A distância da partícula a origem $\boldsymbol{O}$ continua sendo igual a $a$. Em seguida a partícula é rodada de um ângulo $\varphi$ em torno do eixo $z$, tendo a posição final em $P_{3} . b$ ) Os vetores $\overrightarrow{\boldsymbol{r}}_{P_{1}}$ e $\overrightarrow{\boldsymbol{r}}_{P_{3}^{\prime}}$ são os vetores posição da partícula em relação a origem $\boldsymbol{O}$ quando ela ocupa as posições $P_{1}$ e $P_{3}^{\prime}$, respectivamente. O vetor $\overrightarrow{\boldsymbol{n}}_{2}$ é perpendicular ao plano que contém os dois vetores posição nas posições inicial $\left(P_{1}\right)$ e final $\left(P_{3}^{\prime}\right)$. 
No caso em que as rotações em torno dos eixos $y$ e $z$ são infinitesimais, $\Delta \gamma \rightarrow 0$ e $\Delta \varphi \rightarrow 0$, respectivamente, os vetores perpendiculares ao plano que contém os vetores de posição inicial e final da partícula, equações (12a) e (14a), por unidade de área, são aproximadamente igual a:

$$
\begin{aligned}
& \frac{1}{a^{2}} \Delta \overrightarrow{\boldsymbol{n}}_{1} \approx-\Delta \gamma \hat{\mathrm{j}}+\Delta \varphi \hat{k} \\
& +\left[\frac{(\Delta \gamma)}{2}\left((\Delta \varphi)^{2}+\frac{(\Delta \gamma)^{2}}{3}\right) \hat{\mathrm{j}}-\frac{(\Delta \varphi)^{3}}{6} \hat{k}\right], \\
& \frac{1}{a^{2}} \Delta \overrightarrow{\boldsymbol{n}}_{2} \approx-\Delta \gamma \hat{\mathrm{j}}+\Delta \varphi \hat{k} \\
& +\left[\frac{(\Delta \gamma)^{3}}{6} \hat{\mathrm{j}}-\frac{(\Delta \varphi)}{2}\left((\Delta \gamma)^{2}+\frac{(\Delta \varphi)^{2}}{3}\right) \hat{k}\right] .
\end{aligned}
$$

Comparando as expressões anteriores dos vetores $\frac{1}{a^{2}} \Delta \overrightarrow{\boldsymbol{n}}_{1}$ e $\frac{1}{a^{2}} \Delta \overrightarrow{\boldsymbol{n}}_{2}$, válidas para rotações infinitesimais, $\Delta \varphi \stackrel{a^{2}}{\rightarrow} 0 \mathrm{e}$ $\Delta \gamma \rightarrow 0$, coincidem até primeira ordem nessas rotações infinitesimais, ou seja,

$$
\begin{aligned}
& \Delta \vec{\Phi} \approx \frac{\Delta \overrightarrow{\boldsymbol{n}}_{1}}{a^{2}} \approx \frac{\Delta \overrightarrow{\boldsymbol{n}}_{2}}{a^{2}} \quad \Longrightarrow \\
& \Longrightarrow \quad \Delta \stackrel{\Phi}{\Phi}^{2} \approx-\Delta \varphi \hat{\mathrm{j}} .
\end{aligned}
$$

A expressão (16b) coincide com o resuldado $10 \mathrm{~b}$ do vetor resultante $\Delta \vec{\Phi}$. O vetor resultante $\Delta \vec{\Phi}$ independe da ordem em que a partícula realiza as rotações infinitesimais, mostrando que ele satisfaz a condição 1 .

No caso de rotações infinitesimais podemos definir o vetor associado a rotações infinitesimais $\Delta \vec{\Phi}$,

$$
\Delta \vec{\Phi} \equiv \Delta \Phi \hat{n}
$$

sendo $\Delta \Phi$ o ângulo infinitesimal entre os vetores posição inicial e final da partícula após a rotação infinitesimal, e $\hat{n}$ é o vetor perpendicular ao plano que contém esses vetores posição da partícula. O sentido do vetor $\hat{n}$ é dado pela da regra da mão direita em que os dedos vão se fechando enquanto percorrem a trajetória do corpo pontual.

Finalmente mostramos que apesar das expressões dos vetores $\frac{1}{a^{2}} \Delta \overrightarrow{\boldsymbol{n}}_{1}$ e $\frac{1}{a^{2}} \Delta \overrightarrow{\boldsymbol{n}}_{2}$ diferirem de ordem cúbica nas rotações infinitesimais $\Delta \varphi$ e $\Delta \gamma$, veja as equações 15a e $15 \mathrm{~b}$, o vetor velocidade angular é o mesmo nas situações descritas nas Figs. 4 e 5 , uma vez que $\Delta t \rightarrow 0$, temos

$$
\begin{gathered}
\vec{\omega}_{1}=\frac{1}{a^{2}} \frac{\Delta \overrightarrow{\boldsymbol{n}}_{1}}{\Delta t}=\left[-\frac{\Delta \gamma}{\Delta t} \hat{\mathrm{j}}+\frac{\Delta \varphi}{\Delta t} \hat{k}\right], \\
\vec{\omega}_{2}=\frac{1}{a^{2}} \frac{\Delta \overrightarrow{\boldsymbol{n}}_{2}}{\Delta t}=\left[-\frac{\Delta \gamma}{\Delta t} \hat{\mathrm{j}}+\frac{\Delta \varphi}{\Delta t} \hat{k}\right] \Longrightarrow \\
\Longrightarrow \vec{\omega}_{1}=\vec{\omega}_{2}=-\frac{d \gamma(t)}{d t} \hat{\mathrm{j}}+\frac{d \varphi(t)}{d t} \hat{k}=\vec{\omega}
\end{gathered}
$$

As correções de ordem cúbicas nas rotações infinitesimais $\Delta \gamma$ e $\Delta \varphi$ vão a zero no limite de $\Delta t \rightarrow 0$, e portanto não contribuem para as expressões dos vetores velocidade angular $\vec{\omega}_{1}$ e $\vec{\omega}_{2}$. O resultado $18 \mathrm{c}$ nos mostra que o vetor velocidade angular $\vec{\omega}$ é bem definido uma vez que ele independe da ordem em que a partícula realiza as rotações infinitesimais.

\section{Conclusões}

Mostramos através de uma construção algébrica que descreve deslocamentos de rotações de uma partícula em planos distintos, que não podemos associar um vetor ao caso em que envolve rotações finitas, como é apresentado comumente nos livros textos [1, p. 65][2]. Aproveitamos o resultado (8), válido para qualquer rotação realizada primeiro em torno do eixo $z$ e depois do eixo $y$, para reobter o resultado conhecido que podemos associar um vetor para descrever as rotações infinitesimais. Aproveitamos para mostrar por que essa representação vetorial para rotações infinitesimais não pode ser estendida para rotações finitas, apesar desta última poder ser alcançada por rotações infinitesimais consecutivas. Desta forma espero estar estendendo a discussão apresentada na referência [1 p. 276]. Mostramos também que os vetores associados a rotações infinitesimais satisfazem a todas as propriedades de vetores. Acredito que essa forma algébrica de apresentar esses resultados permite a todos nós realizar de forma quantitativa a situação física e mostrar como devemos ser críticos ao usar o ferramental que a Matemática nos propicia para descrever a Natureza: verificar se a nossa formulação abstrata realmente descreve o fenômeno físico que desejamos entender. Agora posso dizer que entendi rotações finitas e vetores.

\section{Referências}

[1] H.M. Nussenzveig, Curso de Física Básica: 1-Mecânica (Edgard E. Blucher, São Paulo, 2013), $5^{\mathrm{a}}$ ed.

[2] ] D. Halliday, R. Resnick e K.S. Krane, Fúsica 1 (LTC, Rio de Janeiro, 1996), v.1, $4^{\mathrm{a}}$ ed., p. 219. 